\title{
Neuroimaging-Based Deep Learning in Autism Spectrum Disorder and Attention-Deficit/Hyperactivity Disorder
}

\author{
Jae-Won Song', Na-Rae Yoon', Soo-Min Jang', Ga-Young Lee ${ }^{2}$, and Bung-Nyun Kim ${ }^{1}$ \\ ${ }^{1}$ Department of Child and Adolescent Psychiatry, Seoul National University Hospital, Seoul, Korea \\ ${ }^{2}$ Seoul National University Hospital, Autism and Developmental Disorder Center, Seoul, Korea
}

\begin{abstract}
Deep learning (DL) is a kind of machine learning technique that uses artificial intelligence to identify the characteristics of given data and efficiently analyze large amounts of information to perform tasks such as classification and prediction. In the field of neuroimaging of neurodevelopmental disorders, various biomarkers for diagnosis, classification, prognosis prediction, and treatment response prediction have been examined; however, they have not been efficiently combined to produce meaningful results. DL can be applied to overcome these limitations and produce clinically helpful results. Here, we review studies that combine neurodevelopmental disorder neuroimaging and DL techniques to explore the strengths, limitations, and future directions of this research area.
\end{abstract}

Key Words: Neuroimaging; Neurodevelopmental disorder; Autism spectrum disorder; Attention-deficit/hyperactivity disorder; Deep learning; Review.

Received: May 13, 2020 / Revision: June 8, 2020 / Accepted: June 11, 2020

Address for correspondence: Bung-Nyun Kim, Department of Child and Adolescent Psychiatry, Seoul National University Hospital, 101 Daehak-ro, Jongno-gu, Seoul 03080, Korea

Tel: +82-2-2072-3647, Fax: +82-2-747-2471, E-mail: kbn1@snu.ac.kr

\section{INTRODUCTION}

During the first 12 months of life, various factors such as heredity and environment affect the progression of neurodevelopmental disorders such as autism spectrum disorder (ASD) and attention-deficit/hyperactivity disorder (ADHD) [1]. Several studies have pointed out that in the case of ASD, behavioral signs may appear during the age of 6-12 months [2], but in clinical settings, on average, clear diagnosis is only possible after 4 years of age [3]. In ADHD, diagnostic accuracy in children older than 7 years of age is limited due to difficulties in distinguishing them from children with normal development [4-7]. Therefore, in longitudinal studies, the stability of ADHD diagnosis before 7 years of age has been a problem; up to 50 other studies have concluded that diagnostic stability rate is below $50 \%$ for children under this age [8-11].

To facilitate better course and prognosis of neurodevelopmental disorders, it is important for clinical interventions to precede certain critical periods of brain plasticity development through early and accurate diagnosis. Although early detection of neurodevelopmental disorders is vital, the development of non-invasive, quantitative, and sensitive biomarkers has been insufficient. It would, thus, be valuable to

This is an Open Access article distributed under the terms of the Creative Commons Attribution Non-Commercial License (https://creativecommons.org/licenses/by-nc/4.0) which permits unrestricted non-commercial use, distribution, and reproduction in any medium, provided the original work is properly cited. detect biomarkers (e.g., brain images, blood, genes, pupillometry, etc.) early in development that can differentiate children with neurodevelopmental disorders and those with normal development pathways.

The several potential biomarkers found thus far exist only as sporadic information, and it is difficult to derive meaningful information on diagnosis by collecting them. In order to solve this problem, deep learning (DL) technology can be used to collect biomarker information, allowing early detection of neurodevelopmental disorders in a simple, accurate, timely, and cost-efficient manner.

This review summarizes the latest papers that use DL in the field of neuroimaging in ASD and ADHD research (Table 1). Through our review, the merits and limitations, as well as future directions of neuroimaging-based DL of neurodevelopmental disorders will be brought to light.

\section{DEEP LEARNING}

DL involves training and testing a multi-layered neural network using artificial intelligence (AI) to learn complex structures and achieve a high level of abstraction [12]. It is a machine learning technique which is different from existing techniques in that it can acquire optimal representation through consecutive nonlinear transformation of large amounts of raw data. This feature has strengths in terms of abstraction 
and complexity, enabling accurate visual recognition. In the medical field, it has gained a spotlight in the field of radiology, and is used for classification, diagnosis, risk factor analysis, prognosis, and prediction of treatment response. In the field of neuroimaging, it is used for classification and diag- nosis of neurologic conditions such as stroke, neurodegenerative disorders, and psychiatric disorders [13-18].

DL uses artificial neural networks (ANN), which are modeled on the structure of human neural networks to perform cognitive functions [19]. In a feedforward network, informa-

Table 1. Summary of neuroimaging based deep learning studies

\begin{tabular}{|c|c|c|c|c|c|c|}
\hline Authors (year) & Sample size & Technique & Features & DL architecture & Comparison & Accuracy (\%) \\
\hline \multirow[t]{2}{*}{ Li et al. $(2018)^{23}$} & $\mathrm{HC}=209$ & sMRI & WB voxel-level & Multi-channel & HC vs. ASD & 76.24 \\
\hline & $A S D=55$ & & & CNN & & \\
\hline \multirow[t]{2}{*}{ Li et al. $(2019)^{24}$} & $\mathrm{HC}=215$ & sMRI & ROI (amygdala, & Dilated-Dense & HC vs. ASD & 79.9 to 92.3 \\
\hline & $A S D=61$ & & hippocampus) & U-Net & & \\
\hline \multirow{2}{*}{$\begin{array}{l}\text { Yoo et al. } \\
(2019)^{25}\end{array}$} & $\mathrm{HC}=47$ & SMRI \& DTI \& & WB voxel-level \& & Random forest & HC vs. ADHD & $69.4 \& 77.8$ \\
\hline & $\mathrm{ADHD}=47$ & rfMRI & region-level & $(\mathrm{RF})$ & & \\
\hline $\begin{array}{l}\text { Moberget et al. } \\
(2019)^{26}\end{array}$ & Cohort $=1401^{*}$ & sMRI \& rfMRI & ROI (cerebeilum) & ICA & & \\
\hline \multirow{4}{*}{$\begin{array}{l}\text { Sidhu et al. } \\
(2019)^{27}\end{array}$} & $\mathrm{HC}=124$ & rfMRI \& tfMRI & WB voxel-level & PCA & HC vs. SPR & $>80$ \\
\hline & $\mathrm{SPR}=55$ & & & & HC vs. ADHD & \\
\hline & $A D H D=19$ & & & & HC vs. ASD & \\
\hline & $\mathrm{ASD}=31$ & & & & & \\
\hline \multirow{2}{*}{$\begin{array}{l}\text { Xiao et al. } \\
(2019)^{28}\end{array}$} & $\mathrm{HC}=81$ & rfMRI & WB voxel-level & SAE & HC vs. ASD & 96.26 \\
\hline & $A S D=117$ & & & & & \\
\hline $\begin{array}{l}\text { Aghdam et al. } \\
(2019)^{34}\end{array}$ & $\begin{array}{l}\text { ABIDE I \& } \\
\text { ABIDE } \| \text { data }^{\dagger}\end{array}$ & rfMRI & WB voxel-level & $\mathrm{CNN}$ & HC vs. ASD & 72.73 \\
\hline \multirow{4}{*}{$\begin{array}{l}\text { Deshpande et al. } \\
(2015)^{35}\end{array}$} & & rfMRI & WB voxel-level & FCC ANN & HC vs. ADHD & $90 \& 95$ \\
\hline & & & & & ADHD inattentive & \\
\hline & & & & & vs. ADHD & \\
\hline & & & & & combined & \\
\hline \multirow{3}{*}{$\begin{array}{l}\text { Jung et al. } \\
(2019)^{36}\end{array}$} & $\mathrm{HC}=125$ & rfMRI & WB voxel-level \& & SVM-RFE & HC vs. ASD & 76.3 to 84.1 \\
\hline & $A S D=86$ & & regional-level & & HC vs. ADHD & \\
\hline & $A D H D=83$ & & & & ASD vs. ADHD & \\
\hline $\begin{array}{l}\text { Kuang et al. } \\
(2014)^{38}\end{array}$ & $\begin{array}{l}\text { ADHD-200 } \\
\text { consortium data }\end{array}$ & rfMRI & ROI (PFC) & DBN & HC vs. ADHD & 44.4 to 80.9 \\
\hline $\begin{array}{l}\text { Hao et al. } \\
(2015)^{39}\end{array}$ & $\begin{array}{l}\text { ADHD-200 } \\
\text { consortium data }\end{array}$ & rfMRI & $\begin{array}{l}\text { ROI (PFC, cingulate, } \\
\text { somatosensory, } \\
\text { visual cortex) }\end{array}$ & $\begin{array}{l}\text { Deep bayesian } \\
\text { network }\end{array}$ & HC vs. ADHD & 48.8 to 72.7 \\
\hline $\begin{array}{l}\text { Wang et al. } \\
(2018)^{40}\end{array}$ & $\begin{array}{l}\text { ADHD-200 } \\
\text { consortium data }\end{array}$ & rfMRI & WB regional-level & SVM & HC vs. ADHD & 78.75 \\
\hline \multirow{2}{*}{$\begin{array}{l}\text { Xu et al. } \\
(2020)^{42}\end{array}$} & $\mathrm{HC}=22$ & fnIRS & Temporal variation & LSTM, CNN & TD vs. ASD & 95 \\
\hline & $A S D=25$ & & & & & \\
\hline \multirow{3}{*}{$\begin{array}{l}\text { Xu et al. } \\
(2019)^{43}\end{array}$} & $\mathrm{HC}=22$ & fnIRS & ROI (bilateral inferior & CGRNN & TD vs. ASD & 90 to 92.2 \\
\hline & $\mathrm{ASD}=25$ & & frontal gyrus and & & & \\
\hline & & & temporal lobe & & & \\
\hline \multirow{2}{*}{$\begin{array}{l}\text { Yoo et al. } \\
(2019)^{25}\end{array}$} & Training dataset=83 & DTI & ROI (10 frontal lobe & WEKA & Training dataset & 90.0 to 95.5 \\
\hline & $\begin{array}{l}\text { Independent } \\
\text { dataset }=36\end{array}$ & & $\begin{array}{l}\text { structures and two } \\
\text { major striatal regions) }\end{array}$ & & $\begin{array}{l}\text { vs. Independent } \\
\text { dataset }\end{array}$ & \\
\hline
\end{tabular}

*philadelphia neurodevelopmental cohort, age 8-23 years, ${ }^{\dagger}$ ABIDE I and II: autism brain imaging data exchange I and II. HC: healthy control, ASD: autism sepectrum disorder, ADHD: attention-deficit/hyperactivity disorder, SPR: schizophrenia, MRI: magnetic resonance imaging, sMRI: structural MRI, DTI: diffuse tensor imaging, rfMRI: resting state functional MRI, tfMRI: task-based function MRI, fNIRS: functional near-infrared spectroscopy, WB: whole brain, ROI: region of interest, PFC: prefrontal cortex, ICA: independent component analysis, PCA: principal component analysis, SAE: sparse auto-encoder, CNN: convolutional neural network, FCC ANN: fully connected cascade artificial neural network, SVM-RFE: support vector machine-recursive feature elimination, DBN: deep belief network, SVM: support vector machine, LSTM: long short-term memory, CGRNN: consisting of CNN and GRU, WEKA: waikato environment for knowledge analysis 
tion is propagated in one direction from the input to the output layer, whereas in a recurrent network, past information makes feedback links that can affect the output. This connection allows information to be retained in a neural network in a manner similar to memory, and a model capable of processing data can be constructed.

There are two main stages of DL. The first (training) stage acquires the optimal algorithm to perform the desired task (e.g., classification, diagnosis, prediction, etc.) by learning the available data. In the second (test) stage, an evaluation of whether the trained model can accurately distinguish the newly observed data is conducted [20].

Convolutional neural networks (CNN), which are most commonly used in neuroimaging, were developed as models of the visual cortex to process visual information and are a type of feedforward neural network. CNN consists of three layers: input layer, convolutional layer, and pooling layer. The convolutional layer consists of several feature maps. All artificial neurons in a given feature map are connected to the previous layer of the feature map in the same region (local connectivity), and the locality is distinguished by connections with different weights from feature maps in other regions. Therefore, a feature map can be used as a feature detector to scan a pattern type, called a kernel. The process of making local connections with different weights to form numerous kernels is done automatically within the network and without any interventions. When multiple convolutional layers exist, increasing amounts of abstract visual information can be processed [12].

Currently, in addition to CNN, various models such as Fully Convolutional Network and Dilated-Dense U-Net are used in research [12].

\section{NEUROIMAGING-BASED DEEP LEARNING IN PSYCHIATRY}

In the field of neuroimaging, the interest in generalization of classification using machine learning is on the rise. DL technology is mainly used in psychiatry as a diagnostic model for distinguishing between patient and normal groups, a model for predicting the risk of developing disorders in a high-risk group, and a model for predicting response to a treatment [12]. A number of studies on diagnostic models have been conducted on Alzheimer's disease and mild cognitive impairment, but the number of studies on neurodevelopmental disorders is small.

In one study, classification using machine learning was performed to evaluate large heterogeneous groups of four diseases: ASD ( $n=988), \operatorname{ADHD}(n=930)$, post-traumatic stress disorder $(n=87)$, and Alzheimer's disease $(n=132)$ [21]. In this study, 18 machine learning classifications were applied, and the predictive powers of these classifiers were combined to form one consensus-classifier proposal. The identified functional connection pattern was firmly applied to differences in classification algorithms, age, and data acquisition sites, and was confirmed to have diagnostic predictive ability along with the ability to distinguish statistically significant differences between groups.

Research on neuroimaging-based DL of neurodevelopmental disorders is mainly conducted using magnetic resonance imaging (MRI), functional MRI (fMRI), and functional near-infrared spectroscopy (fNIRS). Most studies focus on evaluating diagnostic accuracy through DL algorithms, and several MRI studies utilize DL to analyze structural differences between brain regions of patient groups and normal development groups to develop biomarkers for early detection.

\section{MAGNETIC RESONANCE IMAGING}

In a 2019 study by Moon et al. [22], 40 machine learning algorithm studies for ASD published between 2007 and 2018 were systematically reviewed and meta-analysis was conducted. Among them, meta-analysis of 12 samples using structural MRI revealed that the integrated sensitivity was 0.83 , specificity was 0.84 , and area under the curve/partial area under the curve (AUC/pAUC) was 0.90/0.83. Based on these results, the authors deemed the accuracy of the machine learning algorithm using structural MRI in ASD diagnosis to be acceptable.

Li et al. [23] applied multi-channel CNN using a patchlevel data-expanding strategy to brain MRI data to predict ASD early in life. A total of 264 brain MRI scans [55 ASD groups, 209 normal control (NC) groups] registered in the National Database for Autism Research (NDAR) were collected and areas of brain structures showing statistically significant differences between the ASD and NC groups were selected (data-driven anatomical landmark identification). When the corresponding region was extracted from MRI scans and subjected to DL using a multi-channel CNN, the diagnostic accuracy improved by $24 \%$ over a three-dimensional CNN.

Li et al. [24] also used a new DL approach, the DilatedDense U-Net, to find ASD neurobiological anomalies and confirm critical timing of the progression through longitudinal studies of amygdala and hippocampus subfield development. They collected 276 longitudinal brain MRI scans (30 ASD, 31 mild autism spectrum, 215 NC groups) registered in the NDAR. Cross-analysis revealed that the ASD group showed bilateral amygdala and bilateral CA1-3 size expansion at 6 months after birth and bilateral amygdala, left CA1- 
3, left subiculum size expansion at 24 months after birth. Longitudinal analysis showed that at about 6 months of age, there was a size expansion of bilateral amygdala and bilateral CA1-3 in the ASD group with an increase in the size difference between the groups at around 24 months of age. They suggested that overgrowth of amygdala and CA1-3 starting at 6 months of age may be associated with the expression of ASD, and highlighted the possibility of early ASD detection from 6 months of age onwards.

Yoo et al. [25] confirmed that machine learning using multimode neurological imaging and genetic data could distinguish typically developing children from an ADHD group. Polygenetic risk scores calculated from cortical morphology, diffuse scalar and resting state functional connectivity, and norepinephrine/dopamine/glutamate genes were extracted from $47 \mathrm{ADHD}$ children and 47 matched NC children. A model consisting of cortical thickness and volume characteristics achieved a maximum accuracy of $85.1 \%$. Morphological changes across insula, sensory/motor cortex, and ventral frontal cortex were also identified as key predictors, accounting for $18 \%$ of the ADHD measurement scale. Dynamic zone homogeneity within the default network accounted for $6.4 \%$ of the missing errors in the continuous performance test (CPT) and validity testing achieved an accuracy of $69.4 \%$. The results of this study indicate that structural abnormalities related to salience detection, sensory processing and response suppression can be powerful classifiers for ADHD diagnosis and symptoms.

Evidence is accumulating on the role of the cerebellum in psychiatric diseases such as schizophrenia, bipolar disorder, depression, anxiety disorder, and ADHD; however, little is known about its role during the development of these diseases. Currently, it is unclear whether the morphological aspects of the cerebellum are associated with the expression of specific symptoms. In one study, analysis using machine learning was conducted to determine whether morphological characteristics of the cerebellum could reliably predict general cognitive function and psychiatric symptoms in a large-scale developmental community composed of adolescents [26]. The morphological characteristics of the cerebellum were correlated with both general cognitive function and general psychopathology (mean correlations between predicted and observed values: $\mathrm{r}=0.20$ and $\mathrm{r}=0.13$, respectively; $\mathrm{p}<0.001$ ). Analysis of specific symptom areas revealed that norm-violating behaviors ( $\mathrm{r}=0.17 ; \mathrm{p}<0.001)$, psychotic symptoms $(\mathrm{r}=$ $0.12 ; \mathrm{p}<0.001)$ and anxiety symptoms $(\mathrm{r}=0.09 ; \mathrm{p}=0.012)$ were significantly associated with cerebellar morphology. On the contrary, there were no associations with ADHD, depressive disorder, mania, and obsessive-compulsive disorder symptoms. Cerebellar features appeared to be most important in predicting general psychopathology, psychotic symptoms, and norm-violating behaviors.

\section{FUNCTIONAL MAGNETIC RESONANCE IMAGING}

fMRI measures neural activity using blood oxygenationlevel dependent (BOLD) differences. A 2019 meta-analysis study conducted on $\mathrm{AMRI}$ and machine learning algorithm studies on ASD published between 2007 and 2018 [22] found the integrated sensitivity to be 0.69 , specificity to be 0.66 , and AUC/pAUC to be 0.71 .

In one study, nonlinear dimensionality reduction (Locally Linear Embedding) was introduced to extract neural activity information using BOLD time-series [27]. The methodology was verified by leave-one-out-cross-validation (LOOCV) accuracy for classification of psychiatric diagnosis using resting state and task-related fMRI. Embedded fMRI showed high diagnostic performance ( $>80 \%$ ) for 11 datasets including patients with schizophrenia, ADHD, ASD, and NC. Unlike the original fMRI data, embedded fMRI showed significantly better results than chance classification in ten out of 11 datasets.

Xiao et al. [28] attempted to validate diagnostic classification using DL on the full brain frequency from resting-state fMRI data of 198 school-aged ASD children. The average diagnostic accuracy was found to be $96.26 \%$, sensitivity to be $98.03 \%$, and specificity to be $93.62 \%$. These results show higher diagnostic accuracy of up to $15 \%$ when compared with previous studies that classified diagnosis using only one or two frequency bands [29-33].

Aghdam et al. [34] examined whether diagnostic discrimination is possible when resting-state fMRI data of 5-10 year old ASD children is subjected to DL with CNN [34]. Analysis using various methods showed that the maximum diagnostic accuracy was 0.7273 , sensitivity was 0.712 , and specificity was 0.7348 .

In a 2015 study, Deshpande et al. [35] applied a fully connected cascade ANN to compare functional connectivity between ADHD and NC children. Using this method, ADHD children of inattentive and combined subtype and an NC group were classified with an accuracy of $90 \%$, and two subtypes with an accuracy of $95 \%$.

In another fMRI study using machine learning technology, functional connection patterns were examined in ADHD, ASD, and matched typically developing children (7-15 year old males) [36]. In the ASD group, functional connectivity within the limbic system and somatomotor network was increased as compared to the NC group. In addition, it was confirmed that the ADHD group showed an increase in func- 
tional connectivity within the limbic system, visual, default mode, somatomotor, dorsal attention, fronto-parietal, and ventral cognitive networks as compared to the ASD group. Using machine learning, ASD and normal groups, ADHD and normal groups, ADHD and ASD groups were classified with accuracies of $76.3 \%, 84.1 \%$, and $79.3 \%$, respectively.

The ADHD-200 consortium provides open-data sharing of ADHD patients with 776 resting-state fMRI and anatomical datasets aggregated across eight independent imaging sites for better understanding of the neural basis of ADHD. Several studies have been conducted using the ADHD-200 consortium data. Milham et al. used DL to analyze the brain areas that distinguish $\mathrm{NC}$ groups from $\mathrm{ADHD}$ groups, and found that the prefrontal, cingulate, and visual cortices are the most informative [37]. In a study using the same data, it was found that the prefrontal cortex region could be used to distinguish between the ADHD and NC groups of three different samples with accuracies of $44.4 \%, 55.6 \%$, and $80.9 \%$, respectively [38]. In a 2015 study, the dataset of prefrontal, cingulate, somatosensory, and visual cortices were combined to distinguish between the ADHD and $\mathrm{NC}$ groups of three different samples with accuracies of $48.8 \%, 54 \%$, and $72.7 \%$, respectively [39]. In a 2018 study by Wang and Jiao [40], data from the ADHD-200 consortium was used to construct a diagnostic model based on support vector machines (SVMs). The performance of the diagnostic model was analyzed using LOOCV and 10-fold cross-validations (CV). LOOCV revealed that the model had $78.75 \%$ accuracy, $76 \%$ sensitivity, and $80.71 \%$ specificity. The 10 -fold CV average prediction accuracy was $75.54 \pm 1.34 \%$, average sensitivity was $70.5 \pm 2.34 \%$, and mean specificity was $77.44 \pm 1.47 \%$. The differential pattern of ADHD was investigated using SVM, and the result was consistent with those of the previous studies, highlighting its potential as a biomarker.

\section{FUNCTIONAL NEAR-INFRARED SPECTROSCOPY}

It is known that the ASD patient and normal development groups differ in terms of spontaneous hemodynamic fluctuation in oxygenated hemoglobin ( $\mathrm{HbO} 2)$ and deoxyhemoglobin (Hb) [41].

According to a study by Xu et al. [42], hemodynamic changes in the temporal cortex of ASD children show weaker stationarity and stronger memory and persistence for wireless shock than typically developing children. When analyzing whether diagnostic differentiation can be accomplished by applying a DL model to the spontaneous hemodynamic fluctuation of the brain over time, the temporal change of $\mathrm{HbO} 2$ showed $91.4 \%$ specificity, $98.6 \%$ sensitivity, and 95\% accuracy.
The temporal change of $\mathrm{Hb}$ was able to discriminate between the groups with $94.3 \%$ specificity and $97.1 \%$ sensitivity.

In a study by Xu et al. [43], fNIRS was used to measure $\mathrm{HbO} 2, \mathrm{Hb}$, and total hemoglobin in the bilateral inferior frontal gyrus and bilateral temporal cortex for 480 seconds. The record was divided into 7-second units that were partially overlapped and they examined whether ASD and typically developing children could be differentiated using a convolutional gated recurrent neural network (CGRNN), a multi-layer neural network that combines $\mathrm{CNN}$ and gate recurrent unit. As a result, $\mathrm{Hb}$ of channel 10 showed $92.2 \%$ accuracy, $85 \%$ sensitivity, and $99.4 \%$ specificity and $\mathrm{HbO} 2$ of channel 43 showed $90.8 \%$ accuracy, $87.9 \%$ sensitivity, $93.8 \%$ specificity. The total hemoglobin of channel 25 was able to discriminate between the ASD and NC groups with $90 \%$ accuracy, $81.6 \%$ sensitivity, and $98.5 \%$ specificity. Xu et al. suggested that the CGRNN model used in the study can be applied clinically in a mere 7 seconds.

\section{MULTIMODAL APPROACHES}

In a study that used DL, Yoo et al. [44] sought to determine if pre-treatment demographics, clinical symptoms, environmental factors, neuropsychological characteristics, genetic factors, and brain imaging characteristics could predict sleep side effects after methylphenidate (MPH) administration. They collected information on 83 ADHD patients, such as demographics, ADHD Rating Scale-IV (ADHD-RS) and Disruptive Behavior Disorder rating scales, neuropsychological tests [CPT, Stroop color word test], genetic and environmental variables (dopamine and norepinephrine receptor gene [DAT1, DRD4, ADRA2A, SLC6A2] polymorphisms, blood lead, and urine cotinine levels), and structural connectivity of fronto-striatal circuits. The resulting data were analyzed using three machine learning algorithms [logistic ridge regression (LR), SVM, J48]. The accuracy of predicting sleep problems was $95.5 \%$ for LR (AUC 0.99), 91\% for SVM (AUC 0.85 ), and $90 \%$ for $J 48$ (AUC 0.87). Inattentive symptoms in ADHD-RS, CPT response time variability, DAT1, ADRA2A, DRD4, SLC6A2 A3081T polymorphism and fronto-striatal structural connectivity were identified as the most differential features. Validation analysis showed an accuracy of $86.1 \%$ (AUC 0.92). This study suggested that multimodal classifiers, especially neuroimaging features, are helpful in predicting MPH side effects in ADHD children.

\section{DISCUSSION}

Machine learning and DL are among the most popular technologies of recent years, and meaningful results are constant- 
ly being derived by combining them with neuroimaging. They have the advantage of overcoming limitations of traditional analytical methods by using previous mass-univariate statistics and enabling analysis of multivariate brain patterns and inter-regional correlations [45]. As described above, DL techniques provide significant diagnostic accuracy and results in detecting brain regions associated with disease, moving a step forward from traditional neuroimaging techniques. As multimodal approaches become easier with DL, opportunities to better implement the bio-psycho-social model of psychiatric disorders in clinical situations have opened up.

The combination of neuroimaging and DL is still a field that requires much caution and improvement. The first issue is the problem of overfitting. Overfitting refers to the problem of failing to form an appropriate algorithm due to excessive generalization that results from fitting to various types of data [46-48]. This is especially troublesome when the dataset is small [49], causing overestimations of accuracy [50-52]. The more features one needs to analyze, the more training data is required to avoid overfitting, further exacerbating the "curse of dimensionality." Thus, there is a need to apply dimensionality reduction techniques such as principal components analysis or factor analysis in many machine learning studies [52]. In an attempt to overcome this difficulty, some studies have used methods such as regularization [53], early stopping [54], and drop out. [55]

The second issue is that, when using DL, bigger datasets result in more accurate algorithms, implying that the entire dataset will include all the images acquired in different ways (e.g., imaging tool, sites, etc.). In such cases, inadequate standardization can lead to inaccurate processing [56]. As a potential solution to this problem, a field called "brittle AI" is being studied and implemented.

The third problem is that of psychiatric classification. The diagnosis of psychiatric disorders is based on observed symptoms and signs, which can be an obstacle to prediction through DL. The training criteria for evaluating the accuracy of results obtained through DL also follow the current diagnostic classification system. Therefore, a new approach called the research domain criteria initiative [57] was devised to overcome the heterogeneity of psychiatric disorder. This is a method used to detect the brain circuits associated with shared symptoms and derive meaningful results for pathophysiology and etiology of psychiatric illnesses.

Finally, there is an overall lack of transparency and consequent interpretability from such experiments. Unlike general statistical analysis, it is difficult to intuitively recognize the results of analysis from the original data [58]. For example, it may be more difficult to detect abnormalities in specific brain regions of images from individuals using DL. This may be due to a focus on features that are not clinically meaningful (e.g., artifacts) or a lack of information on treatment and/or developmental stages. Input modification methods [59] and deconvolution methods [60] are typically used to address this issue.Research that applies DL to neuroimaging analysis has been mainly conducted over the past 5 years and the field is still at an early stage. There are many research methods, such as targeting the entire brain area or a specific brain region, and each study utilizes various ANNs. Hence, there is great potential for development of the technique. In the future, after overcoming the above-mentioned limitations, DL can be used to discover a biomarker that distinguishes various psychiatric disorders and not just to distinguish between patient and NC groups. In addition, studies on the prediction of prognosis and treatment effects for neurodevelopmental disorders would be beneficial.

\section{CONCLUSION}

Neuroimaging studies of neurodevelopmental disorders have suggested various biomarkers; however, there are limitations in comprehensively analyzing such biomarkers to derive clinically significant results. Considering that the comorbidities of neurodevelopmental disorders are high, a new approach called DL can be applied to the clinical information extracted from currently available standardized diagnostic evaluations to improve the accuracy of diagnosis and better help clinicians in determining diagnosis and treatment.

\section{Acknowledgments}

This work was supported by the National Research Foundation of Korea (NRF), funded by the Ministry of Science, ICT (NRF-2015M3 C7A1028926 to B.-N.K).

\section{Conflicts of Interest}

The authors have no potential conflicts of interest to disclose.

\section{Author Contributions}

Conceptualization: Jae-Won Song, Na-Rae Yoon, Bung-Nyun Kim. Data curation: all authors. Investigation: all authors. Project administration: Bung-Nyun Kim. Supervision: Bung-Nyun Kim. Validation: Bung-Nyun Kim. Writing — original draft: Jae-Won Song, Na-Rae Yoon, Soo-Min Jang, Ga-Young Lee. Writing_review \& editing: Bungnyun Kim.

\section{ORCID iDs}

$\begin{array}{ll}\text { Jae-Won Song } & \text { https://orcid.org/0000-0002-3109-3794 } \\ \text { Na-Rae Yoon } & \text { https://orcid.org/0000-0002-5415-4617 } \\ \text { Soo-Min Jang } & \text { https://orcid.org/0000-0003-3515-7211 } \\ \text { Ga-Young Lee } & \text { https://orcid.org/0000-0002-6687-5737 } \\ \text { Bung-Nyun Kim } & \text { https://orcid.org/0000-0002-2403-3291 }\end{array}$

\section{REFERENCES}

1) Lai MC, Lombardo MV, Baron-Cohen S. Autism. Lancet 2014;383: 896-910. 
2) Filipek PA, Accardo PJ, Baranek GT, Cook EH Jr, Dawson G, Gordon B, et al. The screening and diagnosis of autistic spectrum disorders. J Autism Dev Disord 1999;29:439-484.

3) Baird G, Cass H, Slonims V. Diagnosis of autism. BMJ 2003;327: 488-493.

4) Kadesjö C, Kadesjö B, Hägglöf B, Gillberg C. ADHD in Swedish 3to 7-year-old children. J Am Acad Child Adolesc Psychiatry 2001; 40:1021-1028.

5) Pineda D, Ardila A, Rosselli M, Arias BE, Henao GC, Gomez LF, et al. Prevalence of attention-deficit/hyperactivity disorder symptoms in 4- to 17-year-old children in the general population. J Abnorm Child Psychol 1999;27:455-462.

6) Smidts DP, Oosterlaan J. How common are symptoms of ADHD in typically developing preschoolers? A study on prevalence rates and prenatal/demographic risk factors. Cortex 2007;43:710-717.

7) Campbell SB. Behavior problems in preschool children: a review of recent research. J Child Psychol Psychiatry 1995;36:113-149.

8) Harvey EA, Youngwirth SD, Thakar DA, Errazuriz PA. Predicting attention-deficit/hyperactivity disorder and oppositional defiant disorder from preschool diagnostic assessments. J Consult Clin Psychol 2009;77:349-354.

9) Tandon M, Si X, Luby J. Preschool onset attention-deficit/hyperactivity disorder: course and predictors of stability over 24 months. J Child Adolesc Psychopharmacol 2011;21:321-330.

10) Pierce EW, Ewing LJ, Campbell SB. Diagnostic status and symptomatic behavior of hard-to-manage preschool children in middle childhood and early adolescence. J Clin Child Psychol 1999;28:4457.

11) McGee R, Partridge F, Williams S, Silva PA. A twelve-year followup of preschool hyperactive children. J Am Acad Child Adolesc Psychiatry 1991;30:224-232.

12) Vieira S, Pinaya WH, Mechelli A. Using deep learning to investigate the neuroimaging correlates of psychiatric and neurological disorders: methods and applications. Neurosci Biobehav Rev 2017; 74(Pt A):58-75.

13) Kamal H, Lopez V, Sheth SA. Machine learning in acute ischemic stroke neuroimaging. Front Neurol 2018;9:945.

14) Mateos-Pérez JM, Dadar M, Lacalle-Aurioles M, Iturria-Medina $Y$, Zeighami Y, Evans AC. Structural neuroimaging as clinical predictor: a review of machine learning applications. Neuroimage Clin 2018;20:506-522.

15) Feng R, Badgeley M, Mocco J, Oermann EK. Deep learning guided stroke management: a review of clinical applications. J Neurointerv Surg 2018;10:358-362.

16) Davatzikos C. Machine learning in neuroimaging: progress and challenges. Neuroimage 2019;197:652-656.

17) Zaharchuk G, Gong E, Wintermark M, Rubin D, Langlotz CP. Deep learning in neuroradiology. AJNR Am J Neuroradiol 2018;39: 1776-1784.

18) Plis SM, Hjelm DR, Salakhutdinov R, Allen EA, Bockholt HJ, Long JD, et al. Deep learning for neuroimaging: a validation study. Front Neurosci 2014;8:229.

19) Zhu G, Jiang B, Tong L, Xie Y, Zaharchuk G, Wintermark M. Applications of deep learning to neuro-imaging techniques. Front Neurol 2019;10:869.

20) Chartrand G, Cheng PM, Vorontsov E, Drozdzal M, Turcotte S, Pal CJ, et al. Deep learning: a primer for radiologists. Radiographics 2017;37:2113-2131.

21) Lanka P, Rangaprakash D, Dretsch MN, Katz JS, Denney TS Jr, Deshpande G. Supervised machine learning for diagnostic classification from large-scale neuroimaging datasets. Brain Imaging Behav 2019 Nov 5 [Epub]. https://doi.org/10.1007/s11682-01900191-8.

22) Moon SJ, Hwang J, Kana R, Torous J, Kim JW. Accuracy of machine learning algorithms for the diagnosis of autism spectrum disorder: systematic review and meta-analysis of brain magnetic resonance imaging studies. JMIR Ment Health 2019;6:e14108.

23) Li G, Liu M, Sun Q, Shen D, Wang L. Early diagnosis of autism disease by multi-channel CNNs. Mach Learn Med Imaging 2018; 11046:303-309.

24) Li G, Chen MH, Li G, Wu D, Lian C, Sun Q, et al. A longitudinal MRI study of amygdala and hippocampal subfields for infants with risk of autism. Graph Learn Med Imaging (2019) 2019;11849:164171.

25) Yoo JH, Kim JI, Kim BN, Jeong B. Exploring characteristic features of attention-deficit/hyperactivity disorder: findings from multi-modal MRI and candidate genetic data. Brain Imaging Behav 2019 Jul 18 [Epub]. https://doi.org/10.1007/s11682-019-00164-x.

26) Moberget T, Alnæs D, Kaufmann T, Doan NT, Córdova-Palomera A, Norbom LB, et al. Cerebellar gray matter volume is associated with cognitive function and psychopathology in adolescence. Biol Psychiatry 2019;86:65-75.

27) Sidhu G. Locally linear embedding and fMRI feature selection in psychiatric classification. IEEE J Transl Eng Health Med 2019; 7:2200211.

28) Xiao Z, Wu J, Wang C, Jia N, Yang X. Computer-aided diagnosis of school-aged children with ASD using full frequency bands and enhanced SAE: a multi-institution study. Exp Ther Med 2019;17: 4055-4063.

29) Chen H, Duan X, Liu F, Lu F, Ma X, Zhang Y, et al. Multivariate classification of autism spectrum disorder using frequency-specific resting-state functional connectivity--a multi-center study. Prog Neuropsychopharmacol Biol Psychiatry 2016;64:1-9.

30) Uddin LQ, Supekar K, Lynch CJ, Khouzam A, Phillips J, Feinstein C, et al. Salience network-based classification and prediction of symptom severity in children with autism. JAMA Psychiatry 2013; 70:869-879.

31) Anderson JS, Nielsen JA, Froehlich AL, DuBray MB, Druzgal TJ, Cariello AN, et al. Functional connectivity magnetic resonance imaging classification of autism. Brain 2011;134:3742-3754.

32) Murdaugh DL, Shinkareva SV, Deshpande HR, Wang J, Pennick MR, Kana RK. Differential deactivation during mentalizing and classification of autism based on default mode network connectivity. PLoS One 2012;7:e50064.

33) Iidaka T. Resting state functional magnetic resonance imaging and neural network classified autism and control. Cortex 2015;63:55-67.

34) Aghdam MA, Sharifi A, Pedram MM. Diagnosis of autism spectrum disorders in young children based on resting-state functional magnetic resonance imaging data using convolutional neural networks. J Digit Imaging 2019;32:899-918.

35) Deshpande G, Wang P, Rangaprakash D, Wilamowski B. Fully connected cascade artificial neural network architecture for attention deficit hyperactivity disorder classification from functional magnetic resonance imaging data. IEEE Trans Cybern 2015;45: 2668-2679.

36) Jung M, Tu Y, Park J, Jorgenson K, Lang C, Song W, et al. Surfacebased shared and distinct resting functional connectivity in attention-deficit hyperactivity disorder and autism spectrum disorder. Br J Psychiatry 2019;214:339-344.

37) The ADHD-200 Consortium. The ADHD-200 Consortium: a model to advance the translational potential of neuroimaging in clinical neuroscience. Front Syst Neurosci 2012;6:62.

38) Kuang D, Guo X, An X, Zhao Y, He L. Discrimination of ADHD based on fMRI data with deep belief network. Proceedings of 10th International Conference; 2014 Aug 3-6; Taiyuan, China. Cham, Switzerland: Springer;2014.

39) Hao AJ, He BL, Yin CH. Discrimination of ADHD children based on deep Bayesian network. Proceedings of 2015 IET International Conference on Biomedical Image and Signal Processing; 2015 Nov 19; Beijing, China. 
40) Wang XH, Jiao Y, Li L. Identifying individuals with attention deficit hyperactivity disorder based on temporal variability of dynamic functional connectivity. Sci Rep 2018;8:11789.

41) Zhang F, Roeyers H. Exploring brain functions in autism spectrum disorder: a systematic review on functional near-infrared spectroscopy (fNIRS) studies. Int J Psychophysiol 2019;137:41-53.

42) Xu L, Liu Y, Yu J, Li X, Yu X, Cheng H, et al. Characterizing autism spectrum disorder by deep learning spontaneous brain activity from functional near-infrared spectroscopy. J Neurosci Methods 2020;331: 108538.

43) Xu L, Geng X, He X, Li J, Yu J. Prediction in autism by deep learning short-time spontaneous hemodynamic fluctuations. Front Neurosci 2019;13:1120.

44) Yoo JH, Sharma V, Kim JW, McMakin DL, Hong SB, Zalesky A, et al. Prediction of sleep side effects following methylphenidate treatment in ADHD youth. Neuroimage Clin 2020;26:102030.

45) Calhoun VD, Adali T. Feature-based fusion of medical imaging data. IEEE Trans Inf Technol Biomed 2009;13:711-720.

46) Todd MT, Nystrom LE, Cohen JD. Confounds in multivariate pattern analysis: theory and rule representation case study. Neuroimage 2013;77:157-165.

47) Woolgar A, Golland P, Bode S. Coping with confounds in multivoxel pattern analysis: what should we do about reaction time differences? A comment on Todd, Nystrom \& Cohen 2013. Neuroimage 2014;98:506-512.

48) Alizadeh S, Jamalabadi H, Schönauer M, Leibold C, Gais S. Decoding cognitive concepts from neuroimaging data using multivariate pattern analysis. Neuroimage 2017;159:449-458.

49) Schnack HG, Kahn RS. Detecting neuroimaging biomarkers for psychiatric disorders: sample size matters. Front Psychiatry 2016; 7:50.

50) Kambeitz J, Kambeitz-Ilankovic L, Leucht S, Wood S, Davatzikos C, Malchow B, et al. Detecting neuroimaging biomarkers for schizophrenia: a meta-analysis of multivariate pattern recognition studies. Neuropsychopharmacology 2015;40:1742-1751.
51) Kambeitz J, Cabral C, Sacchet MD, Gotlib IH, Zahn R, Serpa MH, et al. Detecting neuroimaging biomarkers for depression: a metaanalysis of multivariate pattern recognition studies. Biol Psychiatry 2017;82:330-338.

52) Librenza-Garcia D, Kotzian BJ, Yang J, Mwangi B, Cao B, Pereira Lima LN, et al. The impact of machine learning techniques in the study of bipolar disorder: a systematic review. Neurosci Biobehav Rev 2017;80:538-554.

53) Kolbak M, Lauria K, Lee I, Mohan S, Phan HP, Salisbury J. Regularization for deep learning. Deep Learning 2016;221-261.

54) Prechelt L. Early stopping — but when? In: Montavon G, Orr GB, Müller KR, editors. Neural networks: tricks of the trade. 2nd ed. Heidelberg: Springer Berlin Heidelberg;2012. p.53-67.

55) Srivastava N, Hinton G, Krizhevsky A, Sutskever I, Salakhutdinov R. Dropout: a simple way to prevent neural networks from overfitting. J Mach Learn Res 2014;15:1929-1958.

56) Pesapane F, Volonté C, Codari M, Sardanelli F. Artificial intelligence as a medical device in radiology: ethical and regulatory issues in Europe and the United States. Insights Imaging 2018;9:745753.

57) Insel T, Cuthbert B, Garvey M, Heinssen R, Pine DS, Quinn K, et al. Research domain criteria (RDoC): toward a new classification framework for research on mental disorders. Am J Psychiatry 2010; 167:748-751.

58) Suk HI, Lee SW, Shen D; Alzheimer's Disease Neuroimaging Initiative. Latent feature representation with stacked auto-encoder for AD/MCI diagnosis. Brain Struct Funct 2015;220:841-859.

59) Zeiler MD, Fergus R. Visualizing and understanding convolutional networks. Proceeding of 13th European Conference on Computer Vision; 2014 Sep 6-12; Zurich, Switzerland. Cham, Switzerland: Springer;2014.

60) Springenberg JT, Dosovitskiy A, Brox T, Riedmiller MA. Striving for simplicity: the all convolutional net [cited 2020 May 1]. Available from URL: https://arxiv.org/abs/1412.6806. 